\title{
TTR
}

Traduction, terminologie, re?daction

Jean-Marc Gouanvic. Sociologie de la traduction. La science-fiction américaine dans l'espace culturel français des années 1950. Arras, Artois Presses Université, collection "traductologie », 1999.

\section{Judith Lavoie}

Volume 13, numéro 1, 1er semestre 2000

Idéologie et traduction

Ideology and Translation

URI : https://id.erudit.org/iderudit/037400ar

DOI : https://doi.org/10.7202/037400ar

Aller au sommaire du numéro

Éditeur(s)

Association canadienne de traductologie

ISSN

0835-8443 (imprimé)

1708-2188 (numérique)

Découvrir la revue

Citer ce compte rendu

Lavoie, J. (2000). Compte rendu de [Jean-Marc Gouanvic. Sociologie de la traduction. La science-fiction américaine dans l'espace culturel français des années 1950. Arras, Artois Presses Université, collection " traductologie ", 1999.] TTR, 13(1), 195-198. https://doi.org/10.7202/037400ar

Tous droits réservés @ C TTR: traduction, terminologie, rédaction — Les auteurs, 2000
Ce document est protége par la loi sur le droit d'auteur. L'utilisation des services d'Érudit (y compris la reproduction) est assujettie à sa politique d'utilisation que vous pouvez consulter en ligne.

https://apropos.erudit.org/fr/usagers/politique-dutilisation/ 
Jean-Marc Gouanvic. Sociologie de la traduction. La science-fiction américaine dans l'espace culturel français des années 1950. Arras, Artois Presses Université, collection «traductologie», 1999.

Quiconque a été envoûté par les Chroniques martiennes de Ray Bradbury ou a fait un voyage dans le temps grâce à la machine de H.G. Wells sera intéressé par ce livre de Jean-Marc Gouanvic. Postulant le rôle de la traduction comme transformation d'un champ littéraire, l'auteur vise à présenter une sociologie de la traduction de la science-fiction à partir des travaux de Pierre Bourdieu. On aurait pu s'attendre à une étude exclusivement socio-historique ou encore extratextuelle des instances et des causes en présence dans le passage de la science-fiction américaine vers la France, or il n'en est rien. Cette étude se dote d'une double approche : à la fois sociologique et sémiotique. On ne peut que saluer une 
telle entreprise, qui réconcilie deux manières d'aborder la traduction rarement placées côte à côte.

Divisé en huit chapitres, l'ouvrage montre comment se sont opérées l'introduction et la légitimation de la science-fiction américaine en France au cours des années 1950. Mais avant d'aborder ces questions de traduction et d'importation, l'auteur définit ce genre fascinant et troublant qu'est la science-fiction américaine (chapitre I). C'est le Luxembourgeois Hugo Gernsback (1884-1967), aux États-Unis, qui est à l'origine, tant de l'utilisation du terme de science-fiction en 1929, que de l'essor du genre grâce à la création du premier magazine spécialisé de science-fiction, Amazing Stories en 1926. Propulsée par trois auteurs fondateurs (Edgar Poe, Jules Verne et H.G. Wells), la science-fiction américaine se caractérise par deux traits distinctifs : ses thématiques sont fondées sur la nouveauté et une fascination pour l'altérité et le changement, tandis que ses structures éditoriales évoluent de manière autonome, le genre correspondant véritablement à ce que l'auteur appelle une « subculture » (p. 30).

Comment le passage d'un champ littéraire obéissant à ses propres règles s'est-il opéré vers le système français? Comme le démontre Gouanvic, les premières tentatives d'importer le genre en France seront pratiquement vaines (chapitres II et III). Plusieurs raisons expliquent cet échec : une certaine idée que les critiques français se font de la littérature au tournant des années 1920 (par exemple, la littérature jeunesse à laquelle les écrits de Jules Verne sont associés est considérée comme un sous-genre, alors que les traductions en anglais du même Verne ont un énorme succès aux États-Unis à la même époque); on considère également que littérature et science ne font pas bon ménage; enfin, les valeurs de changement technique et social ne sont pas intégrées par le public français avant la Seconde Guerre mondiale.

C'est grâce à l'entreprise menée par les agents Boris Vian, Raymond Queneau et Michel Pilotin que la science-fiction parviendra à s'intégrer en France à partir des années 1950 (chapitres IV et V). Le trio est porté vers la science-fiction américaine précisément pour les raisons qui l'ont empêchée de pénétrer le territoire éditorial français trois décennies plus tôt : elle représente un genre nouveau en alliant le fictif au scientifique et elle subvertit les valeurs sociales et les codes littéraires en vigueur. Vian traduira des nouvelles et des romans, tandis que Pilotin 
(derrière le pseudonyme de Stephen Spriel) rédigera des articles et dirigera la collection "Rayon Fantastique " chez Gallimard/Hachette. Ainsi, la réussite du passage de la science-fiction en France repose non seulement sur la traduction des textes, mais également sur l'importation d'un système éditorial similaire. Voici quelques dates charnières en témoignant : l'année 1951 verra la création de la collection "Rayon Fantastique " conjointement par les éditeurs Hachette et Gallimard; en 1953 sera fondée la revue Galaxie (sorte de clone du magazine américain Galaxy Science Fiction), qui présente une traduction morcelée de la totalité des textes anglais; et, en 1954, les éditions Denoël créeront la collection "Présence du futur ". La principale conséquence de l'importation massive du modèle éditorial américain sera la ghettoïsation du genre en France, comme ce fut le cas aux États-Unis.

On aurait cru, d'emblée, que la difficulté majeure posée par la traduction de la science-fiction serait liée à la présence plus ou moins massive de termes et de syntagmes néologiques, ce que l'auteur appelle, à la suite de Marc Angenot, des " mots-fiction " (p. 77). Or une étude comparative des textes originaux avec leur traduction (chapitre VI) montre que le passage de ces mots-fiction s'est opéré sans trop de perte puisque les deux systèmes linguistiques, anglais et français, empruntent aux mêmes racines grecques et latines en matière de création lexicale. Les technolectes passent donc facilement la rampe : par exemple, le terme anglais Spacers devient en français Spaciens, Subetherics est traduit par Subéthérique, Keratofiber par Kératofibre, et Roboticist par Roboticien (p. 80). Ce sont davantage les référents onomastiques qui, manifestant l'origine étrangère du texte de départ, se démarquent au sein du texte d'arrivée. Comme l'avait démontré Folkart dans Le conflit des énonciations (1991), qu'ils soient ou non signifiants à l'intérieur du texte de départ, les noms propres (de lieu ou de personne) connotent une certaine étrangeté dans le texte d'arrivée du seul fait de leur présence. Le décalage qu'entraîne le maintien de l'onomastique dans les traductions françaises de romans de science-fiction (le Stock Exchange de la ville de New York dans Le choc des mondes par exemple, p. 85) est délibéré en ce qu'il vise à conserver l'origine américaine du texte. D'ailleurs, comme l'explique l'auteur dans son avant-propos, l'emprunt quasi direct du terme de science-fiction en français est symptomatique de la manière dont est importé le genre lui-même, le caractère américain de la science-fiction étant au cœur du projet. Toutefois, la politique traductive de la revue Galaxie se situera aux antipodes de la pratique de l'emprunt (chapitre 
VIII). Les traductions de ce magazine font en effet état d'une adaptation des noms propres fondée sur une homologie phonétique (Tommy Loy devient par exemple Thomas Loyal, Joe Brinkley/Joseph Bordier). Mais, en définitive, qu'on choisisse d'emprunter directement le nom en conservant l'orthographe anglaise ou qu'on l'assimile à une forme française, la présence du traducteur demeure tout aussi tangible.

L'empreinte laissée par Vian sur ses traductions de deux romans d'Alfred E. van Vogt, The World of Null-A (1948) et The Pawns of Null-A (1956), était à prévoir (chapitre VII). Nullement prescriptif (c'est d'ailleurs un trait qui se manifeste dans toutes les analyses de traduction faites par l'auteur), Gouanvic expose les trouvailles de Vian en matière de traduction. Tout en imprimant un ton familier et un style sociolectal à un récit qui en était plutôt dépourvu, Vian-traducteur invente des motsvalises et des néologismes savoureux pour rendre la prose de van Vogt, qu'on pense aux termes stellavion, aéroulotte, distorseur ou encore appareil à similariser (p. 91). La marque unique et originale laissée par Vian sur le texte de van Vogt fait dire à l'auteur, dont le point de vue demeure ouvert et non normatif, que la traduction actualise une lecture possible du texte original.

L'ouvrage se clôt sur ce que l'auteur appelle un " petit plaidoyer pour une sociologie de la traduction ". Disons plutôt qu'il s'agit d'un grand plaidoyer, par ailleurs tout à fait fondé et encore d'actualité, en faveur de la reconnaissance de la discipline traductologique. L'auteur met ici la sociologie de Bourdieu au service d'un questionnement introspectif : où se situent les luttes de pouvoir et quels principes le champ de la traductologie devrait-il adopter pour occuper une place non marginale au sein du monde universitaire? Le débat est ouvert, espérons que les agents évoluant dans le domaine sauront y répondre, tant en actes qu'en paroles.

Judith Lavoie Université de Montréal 